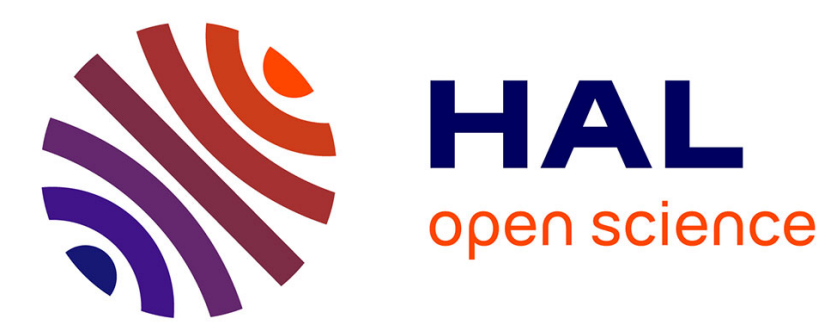

\title{
Designing Social Cognition Models for Multi-Agent Systems through Simulating Primate Societies
}

Sébastien Picault, Anne Collinot

\section{To cite this version:}

Sébastien Picault, Anne Collinot. Designing Social Cognition Models for Multi-Agent Systems through Simulating Primate Societies. 3rd International Conference on Multi-Agent Systems (ICMAS'98), 1998, Paris, France. pp.238-245. hal-00860273

\section{HAL Id: hal-00860273 https://hal.science/hal-00860273}

Submitted on 2 Apr 2019

HAL is a multi-disciplinary open access archive for the deposit and dissemination of scientific research documents, whether they are published or not. The documents may come from teaching and research institutions in France or abroad, or from public or private research centers.
L'archive ouverte pluridisciplinaire HAL, est destinée au dépôt et à la diffusion de documents scientifiques de niveau recherche, publiés ou non, émanant des établissements d'enseignement et de recherche français ou étrangers, des laboratoires publics ou privés. 


\section{Designing Social Cognition Models for Multi-Agent Systems through Simulating Primate Societies}

\author{
Sébastien PICAULT \\ Université Pierre et Marie Curie \\ LIP6 - 4 place Jussieu \\ 75252 Paris Cedex 05, France \\ Sebastien.Picault@lip6.fr
}

\author{
Anne Collinot \\ Université Pierre et Marie Curie/CNRS \\ LIP6 - 4 place Jussieu \\ 75252 Paris Cedex 05, France \\ Anne.Collinot@lip6.fr
}

\begin{abstract}
In this paper, we discuss the advantages of investigating primate societies to build Multi-Agent Systems, and we present our preliminary results in this context. We first give an overview of primates' social competences, then we draw a parallel between the main problems found in the study of primate societies (regarding their social organization) and some of the most commonly encountered issues when designing Multi-Agent Systems. We describe a model of social cognition and perception that we have experimented. Its results show that some social concepts can be implemented by attaching importance to the interactions between the agents, instead of using a complicated individual-based model. Finally, we discuss the main extensions we are working on and propose applications to Multi-Agent technology.
\end{abstract}

\section{Introduction}

Studying primates provides the biologists with good clues to human evolution, clues which do not only concern individual features such as bipedalism or cognitive abilities: it also gives useful examples of complex social competences and behaviours. Section 2 of this paper gives an overview of these competences. In section 3, we show that the study of primate societies is of high interest to us for building Multi-Agent Systems, since there are very similar issues between these two fields. Then, we present in section 4 a reactive model to simulate some primate social competences; its results are discussed in section 5. Finally, section 6 presents our future work and possible extensions to other topics of Multi-Agent Systems.

\section{Primate Social Competences}

In this section, we give an overview of primate social competences, increasing complexity from the recognition of social relations among the group to deception against the conspecifics.

\section{1 "Proto-Concepts"}

Primates live in groups and can recognize each other. Since they remember the outcome of social interactions, the social structure of the group is usually based on a strict hierarchy, almost always linear (i.e. transitive), called the dominance relation. The cognitive specificity of primatesparticularly monkeys and apes - resides on the one hand in their ability to recognize social relations between their conspecifics, and on the other hand in the absence of correlation for the dominance relation with physical differences between individuals. Moreover, primates don't only fight to maintain or modify the social structure: dominant individuals often use threat signals, to which their conspecifics must respond with submission ones.

In fact, using slides-matching methods, Dasser [12, 13] shows that monkeys know which of two individuals has the highest rank, and that they can handle with the transitivity property of the dominance relation. The experiments of Cheney and Seyfarth [9] among vervet monkeys (broadcasting recorded infant calls to several mothers, for instance) demonstrate not only that a mother can identify her offspring, but also that the other present mothers know who, among them, is concerned, because they look at the mother and observe her reactions. This kind of experiments leads to assume that monkeys and apes use "proto-concepts": a very rough symbolic representation of social relations.

Furthermore, the social hierarchy does not (unlike most mammals and the other animals) depend exclusively on physical power. Social factors are more critical to access 
to high ranks in the group: for instance, the rank of the mother (the higher it is, the higher the rank of the offspring will be). It has an influence on the behaviours: for example, Boesch reports that high-rank mothers spend more time bringing up their male offspring than their female one; this discriminating behaviour increases the chances of having a dominant offspring without wasting time (males' rank closely depends on the mother's rank, contrary to females' one). At the opposite, low-rank mothers favour their female offspring, whose rank may raise later, unlike the males.

Another important component of the social structure is the institution of affiliative links and alliance networks between individuals. Monkeys spend a lot of time in grooming each other; however, partners are not randomly chosen: one usually prefers either relatives or previous partners. The probability that one would be solicited correlates with the time he spent grooming the solicitor in the past. This activity has a prophylactic advantage, but its major role is the reinforcement of the social cohesion. It creates very strong links between individuals, so that one will rescue his partner when needed (according to Dunbar [21], gossiping in human groups would play a very close role, with greater efficiency). Depending on the strength of these links and the opening up of the choice of partners, there may be more or less a clannish substructure in the group, with dominance relations between clans.

\subsection{Increasing Social Complexity}

Going further in this overview, we must take a look at other social phenomena, such as "power delusion". Jane Goodall reports the case of an ordinary chimpanzee who had stolen an empty can from the zoologists; this brave deed impressed the whole group, and the thief rapidly became the dominant male, since he only needed to brandish his can to submit his ennemies. Later, the zoologists took back the can, but the thief kept his rank: he won a high social status using deception, but once it has been done, the situation remained stable.

The ability of forming coalitions during fights also allows some monkeys and apes (mostly baboons, macaques, gorillas and chimpanzees) to design complex political behaviours to come to power. De Waal [15] describes the alliances and betrayals between three chimpanzees. The successful strategy is comparable with "tit for tat" (see [2]), since the ally expects the winner to share power with him, as a reward for his support. If not the case, he will wait for an opportunity to revenge.

All the social tensions due to struggle for power could be dangerous for the whole group, since they could lead to perpetual fight. Fortunately, two mechanisms contribute to reducing them: redirecting aggressivity on an unprotected individual, and peacemaking with the loser [16]. By this way, the social structure remains viable.

\subsection{Tactical Deception and "Machiavellian Intel- ligence"}

More recent observations assume that a few species among primates (especially baboons, macaques and the African great apes) have an ability for tactical deception. A lot of anecdotes have been collected by zoologists and compiled (see for instance [6, 7, 4]). First, let us consider an example:

"The juvenile, named Paul, came across an adult female, Mel, just finishing the laborious process of digging up a corm. There were major sources of nutrition at that very dry, cold time of year, but difficult to obtain from the hard ground; Paul was probably unable to dig his own. He looked round, seeing no other baboon, and screamed loudly. His mother, who was higher ranking than Mel, ran into view grunting aggressively and immediately pursued Mel. When they had both left the immediate area, Paul ate the corm." (Byrne [4])

Byrne and Whiten give the following definition for this kind of social rigging:

"Our definition of tactical deception was a functional one: 'acts from the normal repertoire of the agent, deployed such that another individual is likely to misinterpret what the acts signify, to the advantage of the agent'." [4]

Attempting to explain these surprising observations, which might involve intentionality, Byrne and Whiten propose what they call the "machiavellian intelligence" hypothesis: intelligence among primates might have evolved under social pressure. Predation leads the individuals to gather and form groups, but it also implies crowding, tensions and sometimes violence. The "best" strategy in this case would be to learn how to deceive the conspecifics and get maximal selfish benefits from the social structure without harming it or being unmasked by the group. This problematics is very similar to the iterated "Prisoner's Dilemma" when played in an ecological simulation, and to the search of "evolutionarily stable strategies" (see [14, 2]).

In support of this, Dunbar [20] shows that the neocortical ratio correlates with group size among most primate species, indicating that competences in social information processing are critical to maintain group cohesion.

Other theories consider that primate intelligence does not especially emerge from social competences, and of course it is out of purpose to take a decision in that domain. But, to our point of view, it seems that the machiavellian intelligence hypothesis better addresses the problem of which mechanisms allow to build a social organization from interindividual interactions, which typically is very close to DAI issues. 


\section{From Primates to Agents: Problem- Solving among Primates}

In this section, we outlight some features that make primate societies simulation of high interest for Multi-Agent Systems.

\subsection{Primate Societies and Multi-Agent Systems}

We assume that the study of primate societies illustrates very well the difficulties to design Multi-Agent Systems. Here are the three main stages of our approach, which strongly links primate studies to Multi-Agent design.

First, simulating primate societies to help biologists testing their hypotheses would be a natural application of DAI, since the problem involves by itself a group of agents.

The second important step has to deal with the granularity of the agents and their complexity: since primate social competences seem to be very close to ours, we would be led to consider them as a result of high-level cognitive processes - which may be the case, of course - and to build a high-level model. For instance, Byrne [5] proposes a formal notation to describe tactical deception behaviours, based on production rules and symbolic description of domain events: it could be very useful to biologists, but not necessarily to us. In the same way, Worden [28] offers a script-based symbolic learning theory for social behaviours, but says nothing about how the agents build these representations (he only assumes that they have a special cognitive module to do it).

According to Occam's Razor, we prefer, as far as possible, to start with very simple agents, attaching importance to their interactions (instead of their internal structure), to produce complex collective behaviours. We should not increase their individual complexity until such a model proves incomplete.

Third, the mechanisms that will be used for those simulations may be generalized in order to design Multi-Agent engineering principles, or to show that some "reactive" principles (such as stimulus/response based behaviours) are useful in complex social problems.

So, we have to define a radically distributed social model, validate it through simulation, and finally specify general mechanisms for social organization. We try to formulate primate social competences in terms of Multi-Agent Systems, and consequently, we can raise the following issues:

\section{- Proto-concepts}

We have to find a social cognition model that can reproduce the apparently symbolic and logical representations of monkeys. Should we first consider a complicated symbolic model for rank learning, or should we better find a way to reduce the proto-concepts to a lower complexity level, letting them emerge from interactions between the agents?

\section{- Coalitions}

This organization level is quite important regarding Multi-Agent Systems, since it is the basis for team work. Moreover, it could be designed with some mechanisms similar to proto-concepts, if we consider that there are relational proto-concepts (dominance, affiliation, kinship for instance) and organizational ones, concerning coalitions and alliances, which could emerge from the latter.

\section{- Tactical Deception}

It may be necessary to consider some kind of learning or planning. According to biologists, such behaviours cannot be learned by trial-and-error, since they are successful at the first time. They can result either from a progressive evolution (which would have built innate behaviour rules) or from the use of a theory of mind. At the present time, we do not want to pronounce on the minimal cognition model that could produce these behaviours. We believe that the study of the above social phenomena must help us to better specify such a model.

\subsection{An Illustrative Example: the Simulation of Hunting}

We began to work on the simulation of collective red colobus hunting among wild chimpanzees. Boesch describes this activity in [3] and we think that it raises great interest regarding the previous discussed issues.

Nishida [25] also reported hunting behaviours among chimpanzees, but the preys were almost juvenile colobus monkeys, and the behaviour itself, involving a few individuals, seems to be very opportunistic. At the opposite, where Boesch made his observations, the predatory behaviour is more interesting, to our point of view: almost the whole group takes part in hunting adult red colobus monkeys (which provide more meat, but are of course more difficult to catch and kill). In fact, when we analyze the course of the operations, we found that chimpanzees have to deal with some typical Multi-Agent issues:

- Task differentiation: the hunting process begins when the group follows an initiator (often an old chimpanzee), without any other sign. Then, everybody has a role to play: some climb very silently the trees (red colobus monkeys live at the top), some anticipate both colobus and chimpanzee moves to be ready to climb up very quickly and drive the colobus monkeys in the right direction, others run after the preys, others ambush them and catch them. 
- Prey surrounding: the chimpanzees must prevent their preys from escaping, since colobus monkeys, which are smaller than chimpanzees, can easily jump from one tree to another, unlike their predators. It involves on the one hand a very intensive and accurate anticipation of group moves, and on the other hand a global coordination of the whole attacking group.

- Collective learning: from a hunting session to the next one, the distribution of the roles among the group can evolve, depending on individual competences. The problem is not really to know how each chimpanzee learns a particular role, but how the community successfully distributes them, taking the evolution of individual performances into account.

- Individual strategies: the global organization during hunting may be a compromise between a collective goal (maximizing the efficiency of the session) and an individual motivation. For instance, food attribution after the hunting does not depend on social structure (unlike in opportunistic hunting), but on the hunting roles (the catcher gets the maximum). Indeed, some roles are more interesting than others, but to complete hunting, a good repartition must occur, and it does, in fact, without apparent concertation. Moreover, Boesch notices that opportunistic behaviours can be found. There is a visible correlation between number of hunters, success rate, and collaboration; but, the more hunters there are, the more difficult it is to evaluate individual contribution. For this reason, "bystanders" (individuals who were present during the hunting but did nothing) or "latecomer" (who arrived after the capture) may usurp a part of the booty. For a chimpanzee, there is a dilemma between honestly taking part to the hunting and trying to cheat.

All these features make the simulation of hunting an highly challenging application to test parsimony hypotheses regarding social cognition models. More generally, we believe that the study of social cognition and organization should provide powerful models for designing Multi-Agent Systems. In the following section, we report the firsts results of such an approach.

\section{A Reactive Model to Simulate Social Con- cepts}

This section gives an illustration of our approach to the simulation of social competences, which begins with the study of "proto-concepts" (see $\S 2.1$ ). We first present the analysis method we use to describe social interactions. Next, we give a "reactive" model of social cognition. Our work uses a simulation platform called MACACA (see [24]), which concerns the social structure of various macaque societies.

We have kept many features of this platform, such as the simulation framework (based on the same principles than Drogoul's EthoModelling Framework [19]), in which the behaviours of the agents, called tasks, are fixed action patterns, with preconditions. Our preconditions are mostly probabilistic, as well as the issue of the behaviours (for instance, the probability that an agent triggers a fight depends on his motivation in fight, which is an internal stimulus; in the same way, the likelihood of winning depends on the opponents' strength and previous victories).

But, in MACACA, social knowledge is implemented in each agent using a memory of the interactions with the conspecifics: for instance, the outcomes of the conflicts give an indication of dominance relations between an agent and the others. This social information is learned through reinforcement (positive feedback). We believe that this model does not attach enough importance to the interactions between the agents.

\subsection{A Critical Distinction between Organization Levels}

When trying to analyze complex social behaviours such as those found in primate societies, it is very difficult to build a low-level cognition model, since going beyond the simple description of individual behaviours or interactions seems inextricable.

For this reason, we used the Cassiopeia Method (see [11] or [10]) to reduce the complexity of the study. According to its hypotheses, designing Multi-Agent Systems should involve three organization levels: a domain-dependent level, a relational level, and an organizational level.

The agents simultaneously play roles in the three levels. In the context of primate societies, domain-dependent roles concern the physical instantiation of the behaviours (such as Threat, Submit, Eat...); relational roles have to deal with interindividual interactions (like affiliation, dominance or kinship), and organizational roles are involved in coalitions and alliances. Triangular relations (studied for instance by Kummer [23]) between a protector, his "protégé" and an aggressor among many primate societies are in a way very close to this kind of analysis: given these three relational roles, it is easy to draw functional dependencies between them (in terms of affiliation, antagonisms or dominance) leading to the emergence of coalition behaviours.

However, we preferred to focus on determining which cognitive features would be necessary to reproduce protoconcepts in a Multi-Agent simulation, before examining more complex phenomena. 


\subsection{Social Cognition: the Pheromone Model}

We assume that social knowledge (i.e. both relational and organizational knowledges) should be processed in a distributed way, rather than by using individual-based representations. In the MACACA model, everybody knows perfectly his strength relatively to his conspecifics, but nothing about the dominance relation between two of them. Moreover, the positive feedback that builds this social representation concerns only interindividual situations (and not really social ones), so far as the outcome of a relation between two individuals has no effect on their global representations of their conspecifics and cannot be perceived by other individuals.

Another problem occurs when adding a new agent in the population. His social representations have to be built by interacting many times with the others. In natural societies, we might assume that some clues (shiny hair, spatial repartition of agents...), in addition to observation, help the newcomer to build quickly his representations.

Insects lay down or diffuse molecules in the environment to share information about "domain-dependent problems" (such as foraging, nest building, eggs care), and Drogoul $[18,17]$ shows how such mechanisms can be used in problem-solving. The model we propose uses the environment as a social medium, defining "social pheromones" as follows (using the terms defined by the Cassiopeia methodology):

A given social interaction is linked with a particular kind of stimulus, called "social pheromone", which is emitted in the environment; this stimulus, when perceived by an agent, causes a modification in his relational and/or organizational behaviours or knowledges.

By this way, we ensure independence between Cassiopeia levels, and consequently the program modularity.

The purpose of this model is to reproduce the protoconcept phenomena; let us now develop how to use it in the case of the dominance relation. In this context, each agent diffuses a social pheromone (called "rank" for instance), and, instead of memorizing fight outcomes, he maintains sensitivity thresholds to his conspecifics' stimuli (see figure 1). We ensure a direct feedback between the threshold set and the intensity of the emitted stimulus by computing it through the following:

$$
\operatorname{rank}_{i}=\frac{1}{N-1} \sum_{j \neq i} T_{i}(j)
$$

- $r a n k_{i}$ : intensity of the dominance stimulus for agent $i$

- $N$ : size of the population;

- $T_{i}(j)$ : sensitivity threshold of agent $i$ towards dominance stimuli of agent $j$.

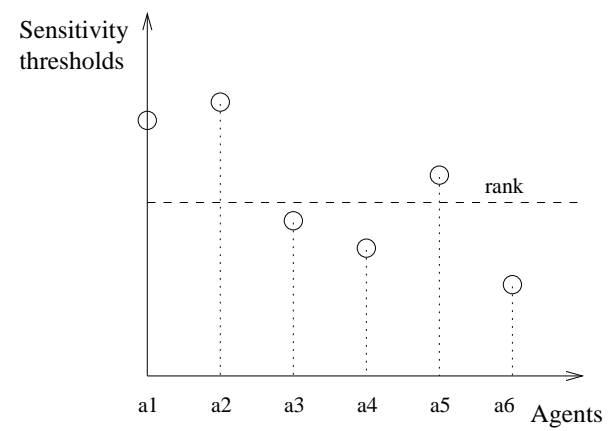

Figure 1. This graph illustrates an agent's social representations of his conspecifics (abscissa). Each agent maintains a set of sensitivity thresholds to determine wether or not he dominates his conspecifics. The average of these thresholds gives the intensity of the agent's own dominance pheromone (called "rank").

When an agent perceives a dominance stimulus, he compares its intensity to the threshold allocated to its emitter, and the result determines the agent's relational behaviour: if the stimulus is low enough, then the agent acts as he would dominate the pheromone's emitter. Therefore, the higher an agent's thresholds are, the less the agent responds to his conspecifics' stimuli; at the same time, his own stimulus has a strong intensity, and probably a great impact on the other agents.

When a domain-dependent role has an influence on relational roles, the thresholds are being adjusted according to the outcome of the interactions. For instance, when a fight occurs, the winner $(W)$ and the loser $(L)$ modify their respective thresholds (see figure 2 and 3): $T_{W}(L)$ increases while $T_{L}(W)$ decreases. The amplitude of the correction is proportional to the former threshold for the loser, and inversely proportional to it for the winner (according to a model proposed in [22]). Therefore, the outcomes of interindividual interactions have an additional influence on the intensity of the winner's and the loser's emitted pheromone, so that their conspecifics may be sensitive to it. That is why this model is a social representation (unlike the former model, which disabled communication), but however distributed (since no individual representation is the mirror of the whole social structure, but the interaction between them makes it emerge).

It is to notice that relational roles can influence each other: for instance, when a female often grooms a dominant male, her rank usually increases. In our model, it means that an affiliative interaction with a high rank-stimulus emitter would lead to increase the partner's dominance thresholds to other individuals, except for this high-rank individual. 


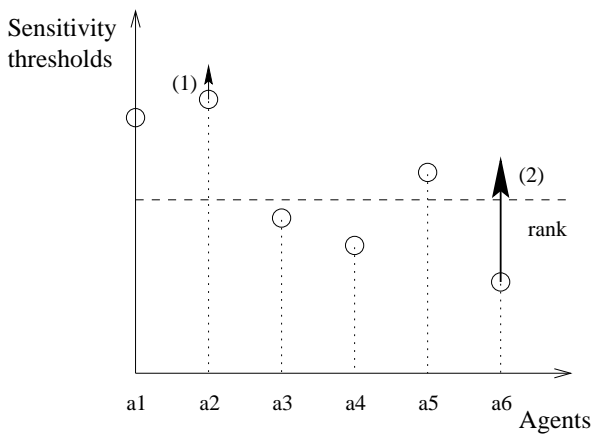

Figure 2. According to the outcome of a fight, the threshold values change. The amplitude of the correction correlates with the former threshold. In the first case (1), the agent has won against a low-rank conspecific (a2), so the habituation to a2's dominance stimulus is low. In the second case (2), the agent has won against a high-rank agent (a6), so the correction (knowledge revision) is quite important.

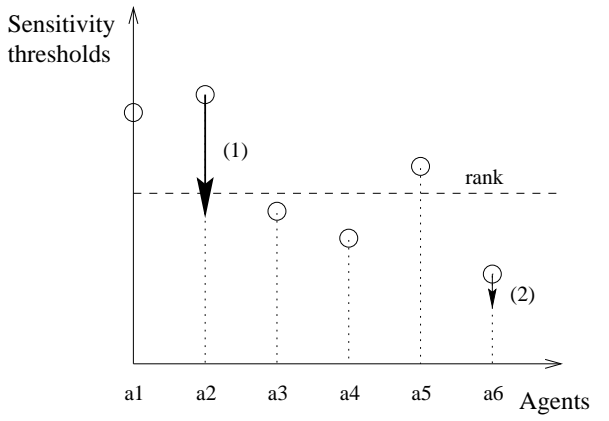

Figure 3. In addition to figure 2, we show here how the thresholds must be corrected when the agents loses against: (1) a low-rank conspecific (a2); (2) a high-rank conspecific (a6).
Finally, we needed to define a perception model to complete our information-sharing one.

\subsection{Simulating the Attention}

According to our pheromone-like model, the perception process uses some of the characteristics that make pheromones powerful:

- They are very simple information bits.

- They are limited in time (they evaporate); therefore, obsolete information is discarded.

- They are limited in space (by diffusion); consequently, the intensity of the stimulus, depending on the distance between the source and the signal, takes part to the meaning of the message.

The first point imposes that the "social pheromone" computer object must be as simple as possible, rather than an intricate negotiation protocol. In fact, a social pheromone is a structure composed with the nature of the relational category (dominance, kinship, affiliation...), the name of the emitter, and the initial intensity. The second point is automatically implemented, since pheromones are being emitted and updated all the time.

For the third point, we defined a relational distance based on the notion of mutual attention, since it seems that, within a well-chosen range, monkeys' social perceptions don't depend on the physical distance. Figure 4 shows two agents with a physical orientation (which is in fact used to move the agents). The angle between the agents and their relative position to each other determine a mutual attention factor. When the agents are face to face, the social distance is minimal, and the mutual attention factor maximal. It is the opposite when they are back to back.

By this way, we assume that our model is coherent with the main characteristics of pheromonal communication. The simulation results we have obtained confirm the validity of this model for proto-concept reproduction.

\section{Results and Discussion}

\subsection{Definitions}

The simulations ran using small populations of agents (from 5 to 30); each simulation took around 40,000 steps (a typical task takes from one to five steps). We measured the evolution of the agents' social representations and a few significative magnitudes (calculated using the frequency of each agent's behaviours), among them: 

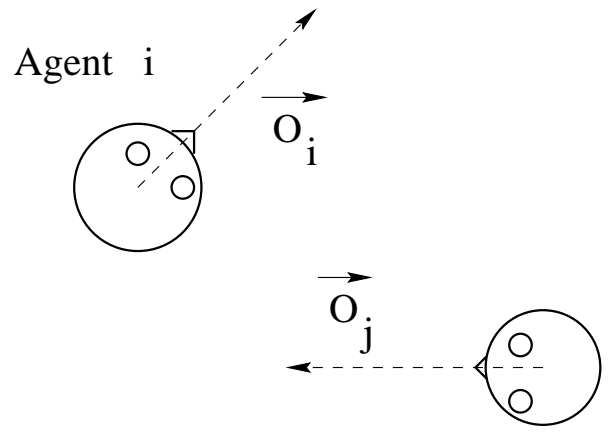

Agent j

\section{Figure 4. Mutual attention: the two agents $i$ and $j$ have orientation vectors; the angle be- tween these two vectors is used to calculate a mutual attention factor, which represents a "social distance".}

- the linearity of the hierarchy, which reflects the structure of the society: close to 1, the global dominance relation is strictly transitive (no triangles); be careful that to this point of view, an agent dominates one of his conspecifics when he wins more fights against him than vice versa - this has nothing to see with the "dominance feeling" of an agent using our perception model. We will call the first case objective dominance, and the other subjective dominance. The objective dominance is a statistical and a posteriori magnitude, while the subjective dominance is a relational response to a stimulus, at a certain point.

- the linearity of the submissions, which indicates wether or not the submission behaviours are used in accordance with the objective dominance. In fact, the agents act using their social knowledge (via task preconditions), so this magnitude gives an estimation of the correlation between subjective and objective dominance.

\subsection{Results and Interpretation}

Using MACACA agents, those two magnitudes usually increase in an exponential way, leading to a strict hierarchy, almost linear, because of an excessive positive feedback effect. At the same time, global aggressivity decreases and affiliation behaviours occur more and more frequently.

With our model, we can observe the cyclic succession of two phases: at the beginning, the two above magnitudes increase exponentially, then they suddenly collapse, before increasing again, and so on. In fact, examining this phenomenon with more details, it has been found that the lin- earity of the submissions always collapses before the linearity of the hierarchy; then, the number of threat behaviours increases to the detriment of affiliative behaviours; finally, more fights occur and the submissions behaviours decrease.

We propose to explain these obervations like follows: imagine two agents with a similar physical power. Their rank stimulus will certainly be quite the same, since they are able to overcome the same conspecifics. But, due to positive feedback, one (called A) may dominate the other (B). Next, if agent B, leaving A, confronts lower-ranking conspecifics, he will win most of the time, and the intensity of his rank stimulus will grow up. When he encounters A again, its intensity may be enough to trigger a response; at the same time, $T_{B}(A)$ remained stable, though B's rank increased. In this situation, each agent's social beliefs (defined as the social behaviour triggered by the perception of a social pheromone) are "erroneous", since they do not correlate with the outcomes of previous conflicts (that explains why the linearity of the submissions collapses first). If a fight occurs, B may win it (in accordance to his rank reinforcement), and both agents will correct their sensitivity thresholds.

In fact, two concepts are modelled: "prejudice" (under the meaning of "erroneous social beliefs" due to unsufficient updating), and "self-confidence" (a positive feedback that does not act directly on the outcome of conflicts but on relational knowledge).

As for proto-concepts, observing the evolution of individual representations leads to the conclusion that the agents act mostly as if they would recognize dominance relations between their conspecifics. Moreover, a new agent can quickly adapt his behaviour to submit himself to the dominant individuals, aggressing at the beginning mostly the weakest ones. Another experiment consists in artificially modifying an agent's threshold; this allows to simulate power delusion effects. In this situation, the doped agent keeps the leadership until another one succeeds in overcoming him.

These results seem to confirm our hypotheses regarding the distinction of organization levels and the relevance of a pheromone-like model to process relational information. In the next section, we will describe some foreseen extensions to this work and possible applications of primate societies simulations to other domains.

\section{Future Work}

We have to extend our work through three directions: first, our social cognition model is to be experimented more widely on other relational roles. Yet we have mostly implemented it for the dominance, affiliative and kinship relations, but these two latter topics have not been enough studied. Moreover, simulating hunting among wild chim- 
panzees could provide new relational and organizational roles. In addition, we assume that organizational roles could be implemented through a similar pheromone-based model (the difficulty would be to define a useful "organizational distance"); this hypothesis is to be confirmed. However, our "mutual attention"-based distance can be extended through the attention towards a common target or goal.

The second point would be to abstract some of our simulation mechanisms to complete the Cassiopeia methodological framework, and to propose some engineering principles which could be implemented into other Multi-Agent Systems. Parunak [26] summarizes studies of natural complex systems, and explains how complex problems should be formulated in terms of a collectivity of simple interacting agents. His examples repose on path planning, brood sorting (among ants), nest building (termites), task differentiation (wasps), flocking (birds and fish) and prey surrounding (wolves). Furthermore, primate societies simulation could provide Multi-Agents tools for relational and organizational issues: social structure building, team work, collective strategies designing, etc. Castelfranchi also points out (in the call for papers of the "Interdisciplinary Workshop on Trust and Deception") the importance of building a general theory of Trust and Deception for Multi-Agent Systems, in order to prevent fraud when individual interests have to compromise with collective goals.

Finally, social phenomena simulation raises stakes that go far beyond biology. In fact, complex behaviours, such as tactical deception, could interest economical or political simulations, since primate behaviours are very close to human ones in this very domain. The simulation of primate social behaviours not only allows to test biological hypotheses and understand collective behaviours, but also has repercussions on other fields in social sciences.

\section{Acknowledgments}

This research is supported by a grant from the French Department of Higher Education and Research.

\section{References}

[1] S. A. Altmann, editor. Social communication among primates. University of Chicago Press, Chicago, 1967.

[2] R. Axelrod and W. D. Hamilton. The evolution of cooperation. Science, (211):1390-1396, 1981.

[3] C. Boesch and H. Boesch. Hunting behaviour of wild chimpanzees in the Taï National Park. American Journal of Physical Anthropology, (78):547-73, 1989.

[4] R. Byrne. The Thinking Ape : Evolutionary Origins of Intelligence. Oxford University Press, 1995.

[5] R. W. Byrne. A formal notation to aid analysis of complex behaviour: Understanding the tactical deception of primates. Behaviour, (127 (3-4)):231-246, 1993.
[6] R. W. Byrne and A. Whiten. Machiavellian Intelligence: social expertise and the evolution of intellect in monkeys, apes and humans. Clarendon Press, Oxford, 1988.

[7] R. W. Byrne and A. Whiten. Tactical deception in primates. The Behavioral and Brain Sciences, (11):233-273, 1988.

[8] C. Castelfranchi and J.-P. Müller, editors. From reaction to cognition, Berlin-Heidelberg, 1995. Springer Verlag, Lecture notes in AI 957.

[9] D. L. Cheney and R. M. Seyfarth. Vocal recognition in freeranging vervet monkeys. Animal Behavior, (30):362-367, 1980.

[10] A. Collinot and A. Drogoul. Using the cassiopeia Method to Design a Soccer Robot Team. Applied Artificial Intelligence. An International Journal, 1997.

[11] A. Collinot, A. Drogoul, and P. Benhamou. Agent-Oriented Design of a Soccer Robot Team. In Tokoro [27].

[12] V. Dasser. A Social Concept of Monkeys. Thèse inaugurale, Université de Zürich, 1987.

[13] V. Dasser. A social concept in Java monkeys. Animal Behavior, (36):225-230, 1988.

[14] R. Dawkins. The Selfish Gene. Oxford University Press, second edition, 1989.

[15] F. B. de Waal. Chimpanzee Politics: power \& sex among apes. Harper \& Row, London, 1982.

[16] F. B. de Waal. Peacemaking among Primates. Harvard University Press, Cambridge, Massachusetts, 1989.

[17] A. Drogoul. When ants play chess (or can strategies emerge from tactical behaviors?). In Castelfranchi and Müller [8], pages 13-27.

[18] A. Drogoul and C. Dubreuil. A Distributed Approach to N-Puzzle Solving. Proceedings of the Distributed Artificial Intelligence Workshop, 1993.

[19] A. Drogoul and J. Ferber. Multi-Agent Simulation as a Tool for Modeling Societies: Application to Social Differentiation in Ant Colonies. Proceedings of the MAAMAW Workshop, 1992.

[20] R. I. Dunbar. Neocortex size as a constraint on group size in primates. Journal of Human Evolution, (20):469-493, 1992.

[21] R. I. Dunbar. Grooming, gossiping and the origins of language. Faber \& Faber, London, 1996.

[22] P. Hogeweg and B. Hesper. The Ontogeny of the Interaction Structure in Bumble Bee Colonies: a MIRROR Model. Behavioural Ecology and Sociobiology, (12):271-283, 1983.

[23] H. Kummer. Tripartite relations in hamadryas baboons. In Altmann [1], 1967.

[24] S. Lalande, A. Drogoul, and B. Thierry. MACACA: a MultiAgent Computer simulation of Animal Communities based on Alliances. Proceedings of Simulating Societies Symposium, 1995.

[25] T. Nishida, S. Uehara, and R. Nyundo. Predatory behaviour among wild chimpanzees of the Mahale Mountains. Primates, (20):1-20, 1979.

[26] H. V. D. Parunak. "Go to the Ant": Engineering Principles from Natural Multi-Agent Systems. Annals of Operations Research, 1997.

[27] M. Tokoro, editor. Proceedings of the Second International Conference on Multi-Agent Systems, Menlo Park, California, 1996. AAAI Press.

[28] R. P. Worden. Primate Social Intelligence. Cognitive Science, (20):579-616, 1996. 